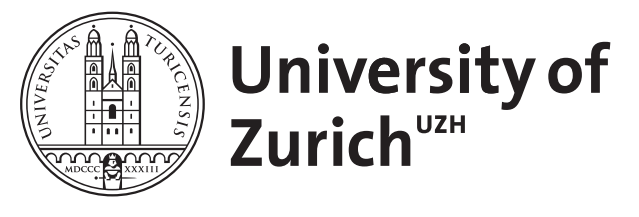

\title{
Evolutionary choice of markets
}

\author{
Gerber, Anke ; Bettzüge, Marc Oliver
}

\begin{abstract}
We consider an economy where a finite set of agents can trade on one of two asset markets. Due to endogenous participation the markets may differ in the liquidity they provide. Traders have idiosyncratic preferences for the markets, e.g.due to differential time preferences for maturity dates of futures contracts. For a broad range of parameters we find that no trade, trade on both markets (individualization) as well as trade on one market only (standardization) is supported by a Nash equilibrium. By contrast, whenever the number of traders becomes large, the evolutionary process selects a unique stochastically stable state which corresponds to the equilibrium with two active markets and coincides with the welfare maximizing market structure
\end{abstract}

DOI: https://doi.org/10.1007/s00199-005-0063-5

Posted at the Zurich Open Repository and Archive, University of Zurich

ZORA URL: https://doi.org/10.5167/uzh-156603

Journal Article

Published Version

Originally published at:

Gerber, Anke; Bettzüge, Marc Oliver (2007). Evolutionary choice of markets. Economic Theory, 30(3):453-472.

DOI: https://doi.org/10.1007/s00199-005-0063-5 


\title{
RESEARCH ARTICLE
}

\section{Anke Gerber · Marc Oliver Bettzüge \\ Evolutionary choice of markets}

Received: 28 July 2004 /Accepted: 9 November 2005 / Published online: 8 December 2005 (C) Springer-Verlag 2005

\begin{abstract}
We consider an economy where a finite set of agents can trade on one of two asset markets. Due to endogenous participation the markets may differ in the liquidity they provide. Traders have idiosyncratic preferences for the markets, e.g. due to differential time preferences for maturity dates of futures contracts. For a broad range of parameters we find that no trade, trade on both markets (individualization) as well as trade on one market only (standardization) is supported by a Nash equilibrium. By contrast, whenever the number of traders becomes large, the evolutionary process selects a unique stochastically stable state which corresponds to the equilibrium with two active markets and coincides with the welfare maximizing market structure.
\end{abstract}

Keywords Endogenous participation - Standardization - Evolution - Stochastic stability

\section{JEL Classification Numbers C79 • G10}

We are grateful to Thorsten Hens, Fernando Vega-Redondo and a referee for valuable comments. We also thank seminar participants at the University of Zurich, the CES research seminar at the University of Munich, the Koc University in Istanbul as well as conference participants at the SAET conference in Ischia, the ESEM in Lausanne and the ESF workshop on Behavioural Models in Economics and Finance in Vienna. A first version of the paper was written while Marc Oliver Bettzüge was visiting the Institute for Empirical Research in Economics at the University of Zurich. Financial Support by the Swiss Banking Institute and by the National Centre of Competence in Research "Financial Valuation and Risk Management" (NCCR FINRISK) is gratefully acknowledged. The NCCR FINRISK is a research program supported by the Swiss National Science Foundation.

\footnotetext{
A. Gerber $(\bowtie)$

Institute for Empirical Research in Economics,

Blümlisalpstrasse 10, 8006 Zurich, Switzerland

E-mail: agerber@iew.unizh.ch
}

M. O. Bettzüge

The Boston Consulting Group,

Im Mediapark 8, 50670 Cologne, Germany 


\section{Introduction}

In standard general equilibrium models without trading frictions all agents can simultaneously trade on all existing market places. In reality, however, it can commonly be observed that agents need to make choices about particular markets they participate in. In the context of financial markets, prominent examples for this kind of decision problem are the choice of an exchange by a broker and by a company issuing shares, or the selection of a set of funds or single assets by an investor.

To analyze the implications of such a situation, this paper studies a simple model with two markets located at the endpoints of an interval, where identical assets can be traded. These markets may differ in two respects. Firstly, given their mean-variance preferences, the traders prefer a liquid market over an illiquid one since it guarantees better predictable price realizations for the assets. ${ }^{1}$ In our model the liquidity of a market increases with the number of traders and hence is endogenous. Whether one market is perceived to be more attractive than the other then depends on the relative size of these markets. Secondly, each trader has an individual preference for one of the two markets. We model this preference by a simple linear cost schedule and assume that traders are sitting at equal distance from each other between the two markets. Hence, agents face a trade-off between the expected liquidity of a market and its characteristics with respect to idiosyncratic preferences. The cost can be given several interpretations, e.g. it may reflect the traders' time preference when the interval represents all possible maturity dates of futures contracts and the positions of the markets represent the tradable maturity dates (see Economides and Siow 1988). Or the cost may reflect a trader's preference or cost of adaption for different information systems or trading platforms used by the exchanges.

As a benchmark case we first study the situation where agents correctly anticipate the liquidity on the two markets. It turns out that a (static) pure strategy Nash equilibrium always exists, but that there may be multiple equilibria including an implausible no trade equilibrium. In particular, there are ranges of the parameter values for this model for which both the situation where all traders meet on one of the two markets (standardization) and the situation where each market is actively used (individualization) coexist as Nash equilibria. This coexistence of equilibria is robust against an increase in the number of traders. By contrast, individualization is the unique welfare maximizing market structure if the number of traders becomes large.

Apart from the multiplicity of equilibria, the static model fails to capture an important element of market selection, especially in the dynamic environment of modern financial markets: Rather than being a one-shot decision, market selection can be regularly revised by market participants based upon the experiences they have made in previous trading periods. Therefore, the paper investigates a dynamic, evolutionary model in which traders are not assumed to have rational expectations about the liquidity on different markets. Instead, the model assumes that agents interact repeatedly and form their expectations on the basis of the observed market

\footnotetext{
1 See O'Hara (1995) for a discussion of the role of liquidity in financial markets.
} 
liquidity in the past. This gives rise to an evolutionary process, where in each period agents play a best reply to a sample of observations made in the past and where they occasionally make a mistake. For this adaptive-play-dynamic (Young 1993) we determine the stochastically stable states, i.e. those states in which the evolutionary process spends most of its time as the error rate goes to zero. We find that there are two critical values for the exogenous costs, such that for costs below the lower value all agents meet on a single market most of the time, while for costs above the upper value both markets remain active. Hence, liquidity considerations lead to a standardization of markets if and only if individual preferences (i.e. the costs in our model) are sufficiently immaterial. In case the two critical values do not coincide, there is a nondegenerate interval of costs for which both, standardization and individualization, are stochastically stable. Different from the static fully rational case, however, this indeterminacy vanishes if the number of traders becomes large. The evolutionary approach predicts that only the situation with two markets will survive in the long run, if the number of traders approaches infinity. Hence, it is the welfare maximizing market structure that is selected for. Moreover, the speed of convergence to the stable market structure is reasonably fast, implying that the evolutionary forces are already effective in the medium run.

The model analyzed in this paper relates to several strands of the literature. It builds on the literature on the selection of markets in the presence of liquidity effects. Important contributions in this field are due to Pagano (1989a,b) and to Economides and Siow (1988). The latter authors, for example, study market selection in a static framework where, as in our model, multiple equilibria with ambiguous welfare properties arise. Our paper goes a step further by analyzing the stability properties of the different equilibria. Similar models are also studied in political economics, where, for example, Alesina and Spolaore (1997) investigate the endogenous determination of the number and size of nations. The model presented in this paper extends this strand of the literature by studying the issue of market selection within an evolutionary framework. Moreover, our paper adds to the recent literature on endogenous participation in financial markets (see Bettzüge and Hens 2001; and chapter 1 in Güth and Ludwig 2000). While we study the evolution of market participation in general, i.e. the choice between different asset markets, these papers concentrate on the evolution of single assets on one market. Hence, our results complement theirs and there are interesting parallels: Bettzüge and Hens (2001) find that incomplete financial markets can be a persistent phenomenon. In Güth and Ludwig (2000) it is shown that there exist stable situations where traders, who are restricted in the number of assets they can trade, do not necessarily exhaust these trading restrictions. By comparison our results show that the existence of two markets need not be a stable situation, if the number of traders is small. Another related paper is Alós-Ferrer and Kirchsteiger (2003) who study the evolution of a market clearing institution vs. non-market clearing institutions. They find that the market clearing institution is always stable but that other, non-market clearing institutions can survive in the long run as well.

The paper is organized as follows. In Section 2 we introduce the static model and derive the set of Nash equilibria. In Section 3 we present the evolutionary approach. We solve for the stochastic stable states and compare them to the welfare maximizing market structures. Finally, in Section 4 we conclude. All proofs are in the Appendix. 


\section{The static economy}

There are $I \geq 4$ agents in our economy who are located at equal distance from each other in an interval that we normalize to $[0,1]$, i.e. agent $i, i=1, \ldots, I$, is located at $(i-1) /(I-1)$. For simplicity we only consider the case where $I$ is even. With a slight abuse of notation by $I$ we also denote the set of agents in our economy. There are two assets, one safe and one risky asset. The safe asset gives a riskless return of $R$ while the risky asset pays a random dividend $d$ with mean $\mu$ and variance $\sigma^{2}$. Every agent is endowed with $\bar{\theta}^{i}=\bar{\theta}+e^{i}$ shares of the risky asset, where $\bar{\theta}$ is a constant and the $e^{i}$ are i.i.d. disturbances with mean 0 and variance $\sigma_{e}^{2}$. Also, each agent is endowed with $\bar{\omega}$ units of the safe asset.

There are two markets where these assets can be traded. Market 1 is located at 0 and market 2 is located at 1 . When trading on market $k(k=1,2)$ agent $i$ determines her demand $\theta^{i}(q)$ for the risky asset such as to maximize a mean-variance utility function, taking the price $q$ of the risky asset as given (the price of the safe asset is normalized to 1). More specifically, agent $i$ solves the following optimization problem

$$
\begin{aligned}
& \max u\left(x^{i}\right)=\mathbb{E}\left(x^{i}\right)-\frac{\gamma}{2} \operatorname{Var}\left(x^{i}\right) \\
& \left(P^{i}\right) \quad \\
& \text { s.t. } x^{i}=\theta^{i} d+R\left(\bar{\omega}+q\left(\bar{\theta}^{i}-\theta^{i}\right)\right) \text { for some } \theta^{i} \in \mathbb{R},
\end{aligned}
$$

where $\gamma>0$ is a measure of the agents' risk aversion, and $\mathbb{E}(\cdot)$ and $\operatorname{Var}(\cdot)$ denote expectation and variance, respectively.

Agents have idiosyncratic preferences for the two markets which we model by a linear cost $c>0$. Trader $i$ 's disutility $c^{i}(k)$ for trading on market $k(k=1,2)$ is given by $c$ times her distance to the market. Hence,

$$
c^{i}(k)=c\left|\frac{i-1}{I-1}-(k-1)\right| .
$$

We assume that agent $i$ 's overall utility from trade is additively separable in the linear cost, i.e. if $x^{i}$ is her final wealth obtained from trade on market $k$, then her utility is

$$
u\left(x^{i}\right)-c^{i}(k) .^{2}
$$

The sequencing of events and actions in our model is the following (see Fig. 1). First, each agent either goes to a market or stays at her position on the line. Then, each agent observes the realization of her endowment, but not the endowments of other agents. An agent who did not go to any of the two markets receives the utility from consuming her endowment. Agents who went to one of the two markets trade assets with other agents on the same market and receive the utility from terminal wealth after trade minus the cost they bear.

2 Alternatively, we can think of the disutility $c^{i}(k)$ as a monetary cost which reduces the final wealth from trade $x^{i}$. In this case, $i$ 's utility from trade on market $k$ is given by $u\left(x^{i}-c^{i}(k)\right)=$ $u\left(x^{i}\right)-c^{i}(k)$ for the particular mean-variance utility function we assume. 
Time

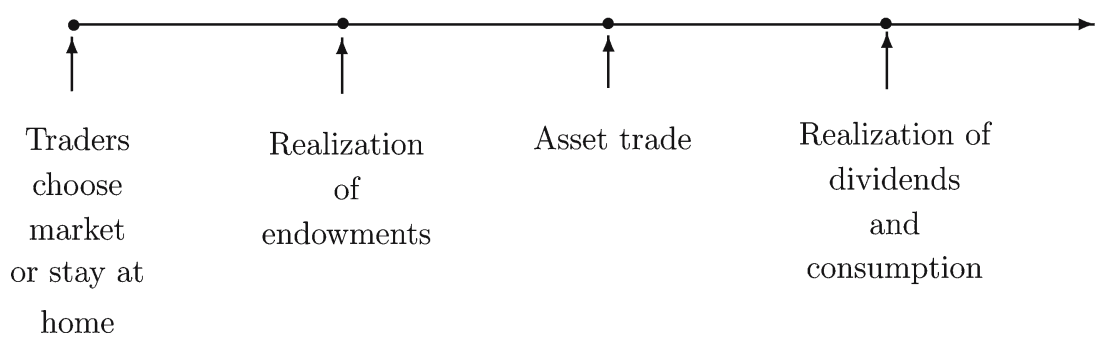

Fig. 1 Timing of events and actions

Observe that the timing is such that agents have to choose a market before knowing their endowments. ${ }^{3}$ What we have in mind are, for example, institutional investors who have to choose a market on behalf of customers whose endowments they do not know yet. ${ }^{4}$ Moreover, we do not allow agents to simultaneously trade on both markets, in other words the traders cannot arbitrage between the markets. This imposes no restriction if, as in one interpretation of our model, the positions of the markets represent different maturity dates for futures contracts and the positions of the traders represent their most preferred maturity dates. In this case arbitrage between the markets is ruled out by physical restrictions. Due to the disutility of trade they face, agents will then trade on at most one market.

We solve the model backwards and first determine an equilibrium on any of the two asset markets taking market participation as given.

\subsection{Equilibrium on the asset market}

Agent $i$ 's optimization problem $\left(P^{i}\right)$ can be rewritten as ${ }^{5}$

$$
\left(\tilde{P}^{i}\right) \max _{\theta^{i}} \mu \theta^{i}+R\left(\bar{\omega}+q\left(\bar{\theta}+e^{i}-\theta^{i}\right)\right)-\frac{\gamma}{2} \sigma^{2}\left(\theta^{i}\right)^{2} .
$$

From the first order condition, which is necessary and sufficient for a solution $\theta^{i}(q)$ of $\left(\tilde{P}^{i}\right)$, we obtain

$$
\theta^{i}(q)=\frac{\mu-q R}{\gamma \sigma^{2}} .
$$

Let $T$ be the set of agents trading on a market. Then, an equilibrium price $q^{*}$ is determined by

$$
\sum_{i \in T} \theta^{i}\left(q^{*}\right)=\sum_{i \in T} \bar{\theta}^{i} .
$$

\footnotetext{
${ }^{3}$ See Pagano (1989b) for a model where actions are taken after the realization of endowments.

${ }^{4}$ For example, brokers buying a seat in an exchange.

${ }^{5}$ Observe that trader $i$ knows her endowment $\bar{\theta}^{i}$ when determining her demand for the risky asset.
} 
Hence,

$$
q^{*}=\frac{1}{R}\left(\mu-\gamma \sigma^{2}\left(\bar{\theta}+\bar{e}_{T}\right)\right)
$$

where $\bar{e}_{T}=\frac{1}{|T|} \sum_{i \in T} e^{i}$. Since the $e^{i}$ are i.i.d., $q^{*}$ is a random variable which depends on the number of agents participating in the market, $|T|$, but not on their identity. It follows that

$$
\theta^{i}\left(q^{*}\right)=\bar{\theta}+\bar{e}_{T}
$$

If we do not take into account the idiosyncratic preferences for trade, then agent $i$ 's ex post utility after trading on the market is given by

$\tilde{U}^{i}\left(q^{*}, e^{i}\right):=\mu\left(\bar{\theta}+\bar{e}_{T}\right)+R \bar{\omega}+\left(\mu-\gamma \sigma^{2}\left(\bar{\theta}+\bar{e}_{T}\right)\right)\left(e^{i}-\bar{e}_{T}\right)-\frac{\gamma}{2} \sigma^{2}\left(\bar{\theta}+\bar{e}_{T}\right)^{2}$,

and her ex ante utility (prior to knowing her endowment and the endowments of the other agents) is

$$
U(T)=U^{i}(T):=\mathbb{E}\left(\tilde{U}^{i}\left(q^{*}, e^{i}\right)\right)=\mu \bar{\theta}+R \bar{\omega}-\frac{\gamma}{2} \sigma^{2} \bar{\theta}^{2}-\frac{\gamma}{2|T|} \sigma^{2} \sigma_{e}^{2},
$$

where we have used the fact that $\mathbb{E}\left(e^{i}\right)=\mathbb{E}\left(\bar{e}_{T}\right)=\mathbb{E}\left(\bar{e}_{T}\left(e^{i}-\bar{e}_{T}\right)\right)=0$ and $\mathbb{E}\left(\bar{e}_{T}^{2}\right)=\sigma_{e}^{2} /|T|$. If we define $U_{0}$ to be the utility from not trading on any of the two markets (i.e. trading on a market with $|T|=1$ ), hence

$$
U_{0}:=\mu \bar{\theta}+R \bar{\omega}-\frac{\gamma}{2} \sigma^{2}\left(\bar{\theta}^{2}+\sigma_{e}^{2}\right)
$$

then

$$
U(T)=U_{0}+K\left(\frac{|T|-1}{|T|}\right)
$$

where $K$ is the constant defined by $K=\gamma \sigma^{2} \sigma_{e}^{2} / 2$. Observe that $U$ is strictly increasing and strictly concave in $|T|$. Let $T_{k}$ be the set of agents trading on market $k, k=1,2$. Then, taking into account the idiosyncratic preferences, $i$ 's ex ante utility for trading on market $k$ with a set of traders $T_{k}$ is given by

$$
U\left(T_{k}\right)-c^{i}(k)
$$

Next we determine the participation at the two markets. 


\subsection{Market participation}

In our economy each trader has three options: she can trade on market 1 or on market 2 or she can stay at home and consume her endowments. In the following we will study the set of pure strategy Nash equilibria for the resulting strategic game.

Let $I$ be the set of players and let $S_{i} \equiv S=\{0,1,2\}$ be the strategy set for player $i$, where 0 means that player $i$ does not trade and $k$ means that $i$ trades on market $k, k=1,2$. For a strategy profile $s \in \prod_{i \in I} S_{i}$ let $T_{k}(s)=\left\{i \mid s_{i}=k\right\}$ be the set of players trading on market $k, k=1,2$, at the strategy profile $s$. For any $i \in I$ trader $i$ 's utility at the strategy profile $s$ is given by

$$
u_{i}(s)= \begin{cases}U_{0}, & \text { if } s_{i}=0, \\ U\left(T_{k}(s)\right)-c^{i}(k), & \text { if } s_{i}=k \in\{1,2\}\end{cases}
$$

Then $\Gamma=\left(I,\left(S_{i}\right)_{i \in I},\left(u_{i}\right)_{i \in I}\right)$ is a standard finite $I$-person normal form game. A strategy profile $s^{*}$ is a (pure strategy) Nash equilibrium of $\Gamma$, if $u_{i}\left(s^{*}\right) \geq u_{i}\left(s_{i}, s_{-i}^{*}\right)$ for all $s_{i} \in S_{i}$ and all $i \in I^{6}$

In a Nash equilibrium agents correctly anticipate their own liquidity effect on a market. This, together with our assumption of price taking behavior on the asset market, introduces an element of bounded rationality on the part of traders. Agents who are aware of their influence on the market size may also be aware of their strategic influence on asset prices. However, a strategic manipulation of asset prices requires knowledge of the price mechanism and hence of other traders' preferences and endowments. At least traders would need to know the distribution of the other agents' characteristics. It seems safe to assume that, in general, they do not have this information in real financial markets. ${ }^{7}$ Hence, we model agents as price takers when they trade on an asset market. Observe that the only rationality requirement then is that agents maximize their utility for given and observed asset prices. They do not have to form rational expectations about future asset prices, since this is a one-period model with short-lived assets and, as usual, our model is silent about how asset prices adjust such as to clear the market. The same is true for the dynamic, evolutionary model we study in the following section, which consists of a sequence of static economies with short-lived assets and no capital accumulation. Even if traders do not know the price mechanism and act as price takers, it is natural to assume that by frequent trading they have learned how the market size influences price volatility and hence ex ante utility. Agents then select the best market given their expectation about the market participation of other traders. In a Nash equilibrium it is assumed that these expectations are correct. As we have argued in the introduction, this rationality assumption may not be realistic and we will abandon it in our evolutionary approach, where we assume that agents play a best reply to simple adaptive expectations.

One immediately verifies that there always exists a trivial Nash equilibrium where there is no trade: If all traders expect everyone to stay at home, then staying at home is indeed a best reply. We will see that depending on the parameters

${ }^{6}$ If $s \in \prod_{i \in I} S_{i}$ is a strategy profile, then by $s_{-i}=\left(s_{1}, \ldots, s_{i-1}, s_{i+1}, \ldots, s_{I}\right)$ we denote the strategy profile for trader $i$ 's opponents (with the obvious adjustment whenever $i=1$ or $i=I$ ).

7 Even if a trader would have this information, she may perceive a proper strategic analysis as too complex. 
there are additional equilibria, one with trade on both markets, where traders split equally among the markets (individualization), and one, where everyone trades on the same market (standardization). Since our aim is to study the trade off between liquidity considerations and costs we restrict our analysis to the set of costs for which standardization is strictly individually rational for all traders. Therefore, we make the following assumption, which implies that $U(I)-c^{i}(k)>U_{0}$ for all $i \in I$ and $k=1,2$ :

Assumption $c<K(I-1) / I$.

Consider the following strategy profiles:

$$
\begin{aligned}
& s^{* 0} \text { with } s_{i}^{* 0}=0 \text { for all } i \in I, \\
& s^{* 1} \text { with } s_{i}^{* 1}=1 \text { for all } i \in I, \\
& s^{* 2} \text { with } s_{i}^{* 2}=2 \text { for all } i \in I, \\
& s^{* 3} \text { with } s_{i}^{* 3}= \begin{cases}1, & \text { if } i \leq I / 2 \\
2, & \text { if } i \geq I / 2+1\end{cases}
\end{aligned}
$$

The following theorem provides a complete characterization of the set of pure strategy Nash equilibria.

\section{Theorem 2.1}

1. If $c<\frac{4 K(I-1)}{I(I+2)}$, then the set of Nash equilibria is given by $\left\{s^{* 0}, s^{* 1}, s^{* 2}\right\}$. $s^{* 1}$ and $s^{* 2}$ are strict Nash equilibria, while $s^{* 0}$ is non strict.

2. If $c \geq \frac{4 K(I-1)}{I(I+2)}$, then the set of Nash equilibria is given by $\left\{s^{* 0}, s^{* 1}, s^{* 2}, s^{* 3}\right\}$. Again, $s^{* 1}$ and $s^{* 2}$ are strict Nash equilibria, while $s^{* 0}$ is non strict. $s^{* 3}$ is a strict Nash equilibrium if and only if $c>\frac{4 K(I-1)}{I(I+2)}$.

As we see there is always a no trade equilibrium but there is also a broad range of costs for which trade on both markets as well as trade on one market only is supported by a Nash equilibrium. Only for small $c$ trade on both markets is not supported as an equilibrium. Observe that the coexistence of equilibria corresponding to standardization and individualization is robust against an increase in the number of traders: The interval for which these equilibria do not coexist becomes vanishingly small if $I \rightarrow \infty$. Hence, the Nash equilibrium concept does not have much predictive power concerning the number of markets in our economy. Intuitively, we may expect individualization to be more stable than standardization if the number of traders is large. In this case the liquidity gain from standardization is small relative to its cost so that it should be more difficult to destabilize the individualization equilibrium than to destabilize the standardization equilibrium. Section 3 will provide an evolutionary analysis which confirms this intuition.

\subsection{Welfare analysis}

Before we proceed with our evolutionary approach we analyze our economy from a welfare theoretic point of view. We again restrict to the case where $c<K(I-1) / I$. 
Obviously, the Nash equilibria of the game cannot be Pareto ranked since there is always an agent who strictly gains and another one who strictly loses when switching from one equilibrium to another. However, we can analyze which market structure would be chosen by a social planner who aims at maximizing a purely utilitarian social welfare function. Since any utility profile corresponds to a particular strategy profile chosen by the agents, the planner's problem is given by ${ }^{8}$

$$
\max _{s \in \prod_{i \in I} S_{i}} W(s)=\sum_{i \in I} u_{i}(s) .
$$

A straightforward computation shows that $\hat{s}$ is a welfare maximizing strategy profile if and only if

$$
\hat{s} \in \begin{cases}\left\{s^{* 1}, s^{* 2}\right\}, & \text { if } c<4 K(I-1) / I^{2}, \\ \left.s^{* 3}\right\}, & \text { if } c>4 K(I-1) / I^{2}, \\ \left.s^{* 1}, s^{* 2}, s^{* 3}\right\}, & \text { if } c=4 K(I-1) / I^{2}\end{cases}
$$

Thus, for small $c$ standardization is welfare maximizing, while for $c$ large individualization maximizes social welfare. Moreover, for all $c$ individualization is welfare maximizing if the number of traders is sufficiently large. This follows from the fact that in a large economy the utility gain from merging two large markets is small relative to the increase in individual costs, so that the welfare maximizing market structure is the one that minimizes costs. Observe that a welfare maximizing strategy profile is always a Nash equilibrium of the game for the range of costs we are considering, but the converse is obviously false.

\section{An evolutionary approach}

We now consider a dynamic version of the static economy analyzed in the last section. Assume that there is a sequence of static economies, which we index by $t=1,2, \ldots$, i.e. the game $\Gamma$ is played repeatedly and in each period $t$ the agents have to decide on which market to trade. Since trade on the markets is anonymous, there are no reputation effects and traders can base their decision on which market to trade only on the observation of the attendance at both markets in previous periods. We assume that traders have to consume all they possess after each trading round so that there is no capital accumulation. Alternatively, we may think of a scenario, where after each trading round all traders die and are replaced by new traders with the same characteristics. Also, assets are short-lived, i.e. they exist only for one period and then are replaced by new assets with the same characteristics.

We now assume that traders, instead of having rational expectations about the participation at the two markets, behave adaptively and play a best reply to the observed market participation in the past. Thus, the rather strong rationality assumption, namely that traders correctly anticipate the size of the two markets, is abandoned in the evolutionary model. ${ }^{9}$ Information is available for the last $m \geq 1$ periods, i.e. the state space $H$ is the set of all histories $h=\left(s^{1}, \ldots, s^{m}\right)$ with $s^{l} \in \prod_{i \in I} S_{i}$ for all $l=1, \ldots, m$. Traders have a limited capacity to process

\footnotetext{
8 Observe that utility is transferable due to the additive separability of costs.
}

9 For a discussion of the behavioral assumptions, see Section 2.2. 
information, or alternatively, gathering information about the previous attendance at the two markets is time consuming and hence costly. We therefore assume that traders can process the information of at most $n \leq m$ periods and, when facing history $h=\left(s^{1}, \ldots, s^{m}\right)$, play a best reply to a sample $\left(s^{r_{1}}, \ldots, s^{r_{n}}\right)$ from $h$. Here, $s_{i} \in S_{i}$ is defined to be a best reply to $\left(s^{r_{1}}, \ldots, s^{r_{n}}\right)$, if it is a best reply to the joint empirical distribution of the other players' actions in the sample:

$$
\frac{1}{n} \sum_{l=1}^{n} u_{i}\left(s_{i}, s_{-i}^{r_{l}}\right) \geq \frac{1}{n} \sum_{l=1}^{n} u_{i}\left(s_{i}^{\prime}, s_{-i}^{r_{l}}\right) \quad \text { for all } s_{i}^{\prime} \in S_{i} .
$$

Observe that in order to determine a best reply in the sense of (1) a trader only needs information about the attendance at the two markets in the sampled periods and in addition she has to recall whether and if so on which market she traded in these periods.

We assume that for a given history $h$ trader $i$ plays strategy $s_{i}$ with positive probability if and only if it is a best reply to some sample of size $n$ from $h$. If all agents simultaneously and independently of each other choose a best reply in this way, then we obtain a Markov process $P^{0}$ on $H$ which is called adaptive play with memory $m$ and sample size $n .{ }^{10}$ Obviously, a state $h$ is absorbing for $P^{0}$ if and only if it is a convention, i.e. $h$ consists of a strict pure strategy Nash equilibrium played $m$ times in a row. It turns out that adaptive play converges to a convention if sampling is sufficiently incomplete.

Theorem 3.1 For any strategy profile $s \in \prod_{i \in I} S_{i}$, let $L(s)$ be the length of the shortest directed path in the best reply graph from s to a strict Nash equilibrium and define $L=\max _{s} L(s) .{ }^{11}$ If $n \leq m /(L+2)$, then adaptive play converges almost surely to a convention.

For the convergence result it is crucial that sampling is sufficiently incomplete since this creates enough stochastic variability in order to prevent the process from getting stuck in cycles. The following example shows that adaptive play may fail to converge if the condition in Theorem 3.1 is not satisfied.

Example 3.1 Let $I=10, K=1, c=9 / 29$ and $m=n=1$. Consider the following strategy profiles $s$ and $s^{\prime}$ with

$$
s_{i}=\left\{\begin{array}{ll}
1, & \text { if } i \leq 4 \\
2, & \text { if } i \geq 5
\end{array} \text { and } \quad s_{i}^{\prime}= \begin{cases}1, & \text { if } i \leq 3 \text { or } i=5 \\
2, & \text { if } i \geq 6 \text { or } i=4 .\end{cases}\right.
$$

Then adaptive play exhibits the cycle $s \rightarrow s^{\prime} \rightarrow s$, more precisely $P_{s s^{\prime}}^{0}=P_{s^{\prime} s}^{0}=1$.

From Theorem 3.1 it immediately follows that adaptive play almost surely converges to the convention corresponding to standardization if $c \leq \frac{4 K(I-1)}{I(I+2)}$, since standardization is the unique strict Nash equilibrium in this case. However,

10 For a formal definition of $P^{0}$ see Young (1993).

11 The best reply graph of $\Gamma$ is given by $(V, E)$, where $V=\prod_{i \in I} S_{i}$ is the set of vertices and $E$ is the set of directed edges such that $\left(s, s^{\prime}\right) \in E$ if and only if $s \neq s^{\prime}$ and there exists a unique player $i$ such that $s_{-i}^{\prime}=s_{-i}$ and $s_{i}^{\prime}$ is a best reply to $s_{-i}$. 
if $c>\frac{4 K(I-1)}{I(I+2)}$, then individualization and standardization are both strict Nash equilibria and convergence may depend on initial conditions. In order to analyze whether in this case convergence to standardization or to individualization is most likely to be observed, we now consider perturbations of the adaptive play process. Such perturbations arise if traders do not always choose a best reply to their observations but occasionally make mistakes or experiment with nonoptimal strategies.

We therefore assume that in each period with probability $\varepsilon>0$ trader $i$ does not play a best reply to some sample of size $n$ but randomly chooses a strategy from $S_{i}$. These mistakes or experimentation activities are independent across traders and independent of the time period $t$. If a trader experiments she chooses any strategy $s_{i}$ with positive probability. The exact specification of these probabilities turns out to be irrelevant. In this way we obtain a perturbed process $P^{\varepsilon}$ which is aperiodic and irreducible for all $\varepsilon>0 .{ }^{12}$ The latter implies the existence of a unique stationary distribution $\mu^{\varepsilon}$ on $H$ satisfying $\mu^{\varepsilon} P^{\varepsilon}=\mu^{\varepsilon}$. A state $h \in H$ is stochastically stable relative to the process $P^{\varepsilon}$ if $\lim _{\varepsilon \rightarrow 0} \mu_{h}^{\varepsilon}>0$. Hence, the stochastically stable states are those states that are most likely to be observed in the long run when the experimentation probability becomes small.

In order to characterize the set of stochastically stable states define a mistake in the transition $h \rightarrow h^{\prime}$ to be a component $s_{i}$ of the right-most element $s$ of $h^{\prime}$ which is not a best reply by agent $i$ to any sample of size $n$ from $h$. Then, for $h, h^{\prime} \in H$ define the resistance $r\left(h, h^{\prime}\right)$ to be the total number of mistakes involved in the transition $h \rightarrow h^{\prime}$ if $h^{\prime}$ is a successor of $h$, otherwise $r\left(h, h^{\prime}\right)=\infty$. For $k=1,2,3$, let $h_{k}=\left(s^{* k}, \ldots, s^{* k}\right)$ be the convention consisting of a repetition of the Nash equilibrium $s^{* k}$. Intuitively, $h_{1}$ and $h_{2}$, i.e. the conventions corresponding to standardization (one market) are stochastically stable if and only if we need (weakly) less mistakes to go from individualization (two markets) to standardization than we need for the opposite direction. Similarly, $h_{3}$, i.e. the convention corresponding to individualization is stochastically stable if and only if we need (weakly) less mistakes to go from standardization to individualization than we need for the opposite direction. For a formal statement of this claim, define $r^{*}$ to be the minimum resistance over all paths from $h_{1}\left(\right.$ or $\left.h_{2}\right)$ to $h_{3}$, i.e.

$$
r^{*}=\min _{\left(h^{1}, \ldots, h^{\tau}\right)} r\left(h^{1}, h^{2}\right)+r\left(h^{2}, h^{3}\right)+\cdots+r\left(h^{\tau-1}, h^{\tau}\right),
$$

where the minimum is taken over all directed paths $\left(h^{1}, \ldots, h^{\tau}\right)$ with $h^{1}=h_{1}$ and $h^{\tau}=h_{3}$. Similarly, define $\tilde{r}$ to be the minimum resistance over all paths from $h_{3}$ to $h_{1}$ (or $h_{2}$ ). We then have the following result.

\section{Lemma 3.2}

$$
\begin{aligned}
h_{1} \text { and } h_{2} \text { are stochastically stable } & \Longleftrightarrow \tilde{r} \leq r^{*}, \\
h_{3} \text { is stochastically stable } & \Longleftrightarrow \tilde{r} \geq r^{*} .
\end{aligned}
$$

In order to see, under which conditions it is true that $\tilde{r}>r^{*}$, assume we are in the convention $h_{3}$, where there is trade on both markets. Then, for going from $h_{3}$ to any convention with trade on one market only, e.g. $h_{1}$, we need a certain number of

\footnotetext{
12 For a formal definition of $P^{\varepsilon}$ see Young (1993).
} 
traders to switch from market 2 to market 1 by mistake. This number has to be sufficiently large, i.e. these traders have to create enough liquidity at market 1 , so that it is a best reply for the remaining players at market 2 to switch as well. The higher the cost $c$, the more liquidity is needed in order to induce a "best reply switch" to the more distant market, i.e. $\tilde{r}$ is non-decreasing in $c$. Conversely, assume we are in convention $h_{1}$, where everyone trades on market 1 . Again a certain number of traders has to switch from market 1 to market 2 by mistake in order to induce a best reply switch to market 2 by the remaining players, who are closer to market 2 than to market 1 . The higher the cost $c$ the more attractive it is for a trader to go the the closest market, in which case less traders are needed, who switch by mistake. In other words, $r^{*}$ is non-increasing in $c$. Hence, if $c$ is large, it is easier to switch from $h_{1}$ or $h_{2}$ to $h_{3}$, and therefore $h_{3}$, the convention with trade on both markets, is stochastically stable. Conversely, if $c$ is small, then $h_{1}$ and $h_{2}$, the conventions with trade on one market only, are stochastically stable. This is the intuition for the following theorem.

Theorem 3.3 Let $I>4 n$ and $n \leq m /(L+2)$. Then there exist $c_{1}^{*}, c_{2}^{*}$ $\in\left(\frac{4 K(I-1)}{I(I+2)}, \frac{K(I-1)}{I}\right), c_{1}^{*} \leq c_{2}^{*}$, such that $h_{1}$ and $h_{2}$ are the unique stochastically stable states if $c<c_{1}^{*}$, and $h_{3}$ is the unique stochastically stable state if $c>c_{2}^{*}$. If $c_{1}^{*}<c_{2}^{*}$, then all states $h_{1}, h_{2}, h_{3}$, are stochastically stable for $c \in\left(c_{1}^{*}, c_{2}^{*}\right)$.

The coexistence of stochastically stable states in the interval $\left(c_{1}^{*}, c_{2}^{*}\right)$ is due to the fact that we only have a finite number of traders, which implies that the resistances $r^{*}$ and $\tilde{r}$ are step functions in $c$. Hence, one can conjecture that the indeterminacy vanishes if the number of traders goes to infinity, which is confirmed by the following theorem.

Theorem 3.4 For fixed $n$, if we write $r^{*}, \tilde{r}, c_{1}^{*}, c_{2}^{*}$, as functions of the number of traders $I$, then there exists $I_{0}=I_{0}(c, K)$ such that

$$
r^{*}(I) \leq n \frac{c+K}{c} \text { for all } I \geq I_{0} .
$$

Moreover,

$$
\lim _{I \rightarrow \infty} \tilde{r}(I)=\infty
$$

and

$$
\lim _{I \rightarrow \infty} c_{1}^{*}(I)=\lim _{I \rightarrow \infty} c_{2}^{*}(I)=0 .^{13}
$$

In order to understand the effect of an increase in the number of traders on the stability of the different conventions consider first the case where the economy is in a state of standardization, where everyone trades on the same market, let's say on market 2 . In order to trigger a transition to the convention with trade on both markets we need a certain number of traders, $F^{*}$, to switch to market 1 by

13 Observe that we cannot fix both $n$ and $m$ and let $I \rightarrow \infty$ since $L$ depends on $I$. From the proof of Theorem 3.1 it follows that $L \leq 2 I$, i.e. $L$ increases with $I$ at most linearly. 
mistake. ${ }^{14}$ This number has to be large enough so that for the marginal trader, who is sitting next to the "mutants", the cost reduction from switching to market 1 is larger than the utility loss she suffers due to the decrease in liquidity. If $I$ goes to infinity the cost reduction converges to $c$, independent of the number of mutants, while the utility loss is bounded above by $K / F^{*}$. Hence, the number of mutants necessary to equate the cost reduction and the utility loss, i.e. to trigger a transition from standardization to individualization is bounded above by $(c+K) / c$ since $F^{*}$ is an integer.

Consider now the case where the economy is in a state of individualization, where there is trade on both markets. Again, in order to trigger a transition to standardization, where, for example, everyone trades on market 1 , we need enough players to switch to market 1 by mistake. The number of mutants has to be large enough so that for the marginal trader sitting next to the mutants the utility gain due to the increase in liquidity is larger than the increase in cost for trading on the more distant market. If $I$ goes to infinity both the cost increase, as well as the utility gain go to zero. The former is due to the fact that for a fixed number of mutants the marginal trader moves closer and closer to the trader in the middle of the interval as $I$ goes to infinity. The utility gain goes to zero because for a fixed number of mutants the difference in liquidity at the two markets has a negligible effect on utility, if $I$ becomes large since marginal utility converges to zero. Moreover, an inspection of the traders' preferences reveals that the utility gain goes to zero at a higher rate $\left(O\left(I^{-2}\right)\right)$ than the increase in cost $\left(O\left(I^{-1}\right)\right)$, i.e. liquidity considerations become relatively unimportant compared to costs. Hence, in order to trigger a transition from individualization to standardization we need more and more mutants if $I$ becomes large. This informal argument shows that we need a much smaller number of mistakes to go from standardization to individualization than we need in the reverse transition, if the economy is large. Hence, trade on both markets with traders splitting equally between the markets is the unique stochastically stable state if $I$ is large.

Theorem 3.4 is an important result. It shows that for all $0<c<K$ and $I$ sufficiently large there is a unique stochastically state which is given by the convention with trade on both markets. ${ }^{15}$ Hence, recalling the result from Section 2.3, in a large economy the evolutionary process selects the welfare maximizing market structure. By way of contrast we have seen that the indeterminacy of Nash equilibria is robust against an increase in the number of traders. Theorem 3.4 also implies that convergence to the market structure with trade on both markets is reasonably fast. ${ }^{16}$ The following theorem provides a bound on the expected waiting time until the process reaches the stochastically stable convention. It shows that in our model the evolutionary forces are already effective in the medium run.

Theorem 3.5 Let $W(h, \varepsilon)$ be the expected number of periods until the convention $h_{3}$ is first reached given that the process $P^{\varepsilon}$ starts in $h$. Then, there exists an $I_{0}=I_{0}(c, K)$ such that for $I \geq I_{0}$ and any $h \neq h_{3}$,

\footnotetext{
${ }^{14}$ For an exact definition of $F^{*}$ see the Appendix. The argument, however, can be made without specifying $F^{*}$.

15 Recall that we assumed $c<K(I-1) / I$ and $\lim _{I \rightarrow \infty} K(I-1) / I=K$.

16 Several authors (see Kandori et al. 1993; Ellison 1993) have pointed out that equilibrium selection theories only give reasonable predictions when the selection is already effective in the medium run.
} 


$$
W(h, \varepsilon)=O\left(\varepsilon^{-n(c+K) / c}\right) \text { as } \varepsilon \rightarrow 0 .
$$

We see that the bound on the expected waiting time is independent of the number of traders. In this sense, evolution in our model can be considered as fast (cf. Ellison 2000).

Finally, we analyze how the stability of the different conventions is influenced by the traders' risk aversion and by the risk present in the economy, namely by the idiosyncratic endowment and the aggregate dividend risk.

Theorem 3.6 Let $I>4 n$ and $n \leq m /(L+2)$. Then the thresholds $c_{1}^{*}$ and $c_{2}^{*}$ are non-decreasing in the coefficient of risk aversion $\gamma$ and in the variances of dividends $\sigma^{2}$ and of endowments $\sigma_{e}^{2}$.

This result is intuitive given our observations concerning an increase in the number of traders. Here, we just get the opposite effect: If traders become more risk averse or if the variances of dividends or endowments increase, then liquidity considerations become more important relative to idiosyncratic preferences and the range of costs for which standardization is stochastically stable becomes larger.

\section{Conclusion}

We have studied the choice of markets in the presence of trading frictions and liquidity effects. While the static model has multiple Nash equilibria including a no trade equilibrium, the evolutionary process selects a unique equilibrium for a large range of costs: For sufficiently low costs, all agents will meet on one market (most of the time), while for sufficiently high costs, there will be trade on both markets (most of the time). Hence, we observe standardization (e.g. of maturity dates or trading platforms) if and only if liquidity considerations are relatively more important than idiosyncratic preferences for the two markets. Different from the static model, the interval of costs, for which standardization as well as trade on both markets (individualization) are stochastically stable, vanishes if the number of traders becomes large. Moreover, our analysis suggests that in economies with a large number of traders we will observe individualization rather than standardization, which is also the welfare maximizing market structure. While evolutionary models are often subject to the criticism that the evolutionary forces are only effective in the ultra-long run, here we are able to show that the convergence to the stochastically stable market structure is reasonably fast.

\section{Appendix: Proofs}

Proof of Theorem 2.1. It is immediate to see that $s^{* 0}, s^{* 1}$ and $s^{* 2}$ are always Nash equilibria, and that $s^{* 1}$ and $s^{* 2}$ are strict, while $s^{* 0}$ is not strict. Let $s^{*}$ be a Nash equilibrium and let $t_{k}:=\left|T_{k}\left(s^{*}\right)\right|$ for $k=1,2$. Assume by way of contradiction that there exists $i$ and $j<i$ such that $s_{i}^{*}=1$ and $s_{j}^{*} \neq 1$. Assume first that $s_{j}^{*}=0$. Then,

$$
\frac{t_{1}}{t_{1}+1} K \leq c \frac{j-1}{I-1}<c \frac{i-1}{I-1}
$$


which is a contradiction since $i$ could improve by choosing $s_{i}=0$ instead of $s_{i}^{*}=1$. Assume next that $s_{j}^{*}=2$. Then,

$$
\frac{t_{2}-1}{t_{2}} K-c \frac{I-j}{I-1} \geq \frac{t_{1}}{t_{1}+1} K-c \frac{j-1}{I-1},
$$

which implies that

$$
\frac{t_{2}}{t_{2}+1} K-c \frac{I-i}{I-1}>\frac{t_{1}-1}{t_{1}} K-c \frac{i-1}{I-1} .
$$

This is again a contradiction since $i$ could improve by choosing $s_{i}=2$ instead of $s_{i}^{*}=1$. In a similar way one shows that $s_{i}^{*}=2$ implies that $s_{j}^{*}=2$ for all $j>i$.

Assume now that there exists $i$ with $s_{i}^{*}=1$. Since $c<K(I-1) / I$, it follows that $K(j-1) / j>c(j-1) /(I-1)$ for all $2 \leq j \leq I$. Hence, $s_{i+1}^{*} \in\{1,2\}$. Similarly, if there exists $i$ with $s_{i}^{*}=2$, then $s_{i-1}^{*} \in\{1,2\}$.

Assume that $s^{*} \neq s^{* 0}$. By the above this implies the existence of some $i^{*}, 0 \leq$ $i^{*} \leq I$, such that $s_{i}^{*}=1$ for $i \leq i^{*}$ and $s_{i}^{*}=2$ for $i \geq i^{*}+1$. Hence, if $i^{*}=0$, then $s^{*}=s^{* 2}$, and if $i^{*}=I$, then $s^{*}=s^{* 1}$. If $1 \leq i^{*} \leq I-1$ it follows that

$$
\begin{aligned}
K \frac{i^{*}-1}{i^{*}}-c \frac{i^{*}-1}{I-1} & \geq K \frac{I-i^{*}}{I-i^{*}+1}-c \frac{I-i^{*}}{I-1} \\
\text { and } K \frac{I-i^{*}-1}{I-i^{*}}-c \frac{I-i^{*}-1}{I-1} & \geq K \frac{i^{*}}{i^{*}+1}-c \frac{i^{*}}{I-1} .
\end{aligned}
$$

If $i^{*}<(I-1) / 2$, then (2) and (3) are equivalent to

$$
\begin{aligned}
\frac{c}{I-1} & \geq \frac{K}{i^{*}\left(I-i^{*}+1\right)} \\
\text { and } \quad \frac{c}{I-1} & \leq \frac{K}{\left(I-i^{*}\right)\left(i^{*}+1\right)} .
\end{aligned}
$$

This implies that $i^{*} \geq I / 2$ which is a contradiction. In the same way we obtain a contradiction in the case $i^{*}>(I+1) / 2$. Hence, if $s^{*} \notin\left\{s^{* 0}, s^{* 1}, s^{* 2}\right\}$ is a Nash equilibrium, then $s^{*}=s^{* 3}$ and $c \geq 4 K(I-1) /(I(I+2))$, where the latter follows from (2) for $i^{*}=I / 2$. Finally, it is immediate to see that $s^{* 3}$ is indeed a Nash equilibrium if $c \geq 4 K(I-1) /(I(I+2))$. The Nash equilibrium is strict if and only if the inequality is strict. This proves the theorem.

Proof of Theorem 3.1. A game $\Gamma$ is weakly acyclic, if from any strategy profile there exists a directed path in the best reply graph of $\Gamma$ to some strict pure strategy Nash equilibrium of $\Gamma$. As Young (1993) (Theorem 1) has shown, for a weakly acyclic game adaptive play converges almost surely to a convention if $n \leq m /(L+2)$. We cannot directly apply this theorem in our context since Young defines a best reply to a sample to be a best reply to the product of the other players' empirical distribution of play, while we define it to be a best reply to the joint empirical distribution of play. However, an inspection of the proof in Young (1993) reveals that it only involves arguments where agents play a best reply to a sample with identical strategy profiles, in which case the two different notions of best replies 
to a sample obviously coincide. Hence, we can use the same proof to show an analogue of Young's theorem for our best reply dynamic.

It remains to prove that our game $\Gamma$ is weakly acyclic. To this end, let $s^{*}$ be an arbitrary strategy profile. First consider the case, where $s^{*}=\bar{s}^{F}$ for some $0 \leq F \leq I$, and $\bar{s}^{F}$ is defined by

$$
\bar{s}_{i}^{F}= \begin{cases}1, & i \leq F \\ 2, & i \geq F+1 .\end{cases}
$$

Without loss of generality let $F \leq I / 2$. If $c \neq 4 K(I-1) /(I(I+2))$, then all Nash equilibria except for the no trade equilibrium are strict. In particular, if $\bar{s}^{F}$ is a Nash equilibrium then it is strict. Hence, if $\bar{s}^{F}$ is a Nash equilibrium we are done. Otherwise, $\bar{s}_{i}^{F}$ is not the best reply to $\bar{s}_{-i}^{F}$ for $i=F$ or $i=F+1$. If $\bar{s}_{i}^{F}=1$ is not the best reply to $\bar{s}_{-i}^{F}$ for $i=F$, then $s_{i}=2$ is a best reply (observe that $s_{i}=0$ cannot be the unique best reply for $i=F$ ). Hence, $\bar{s}^{F} \rightarrow \bar{s}^{F-1}$ in the best reply graph. Since by construction $\bar{s}_{i}^{F-1}$ is a best reply to $\bar{s}_{-i}^{F-1}$ for $i=F$, it follows that either $\bar{s}^{F-1}$ is a (strict) Nash equilibrium or $\bar{s}_{i}^{F-1}$ is not a best reply to $\bar{s}_{-i}^{F-1}$ for $i=F-1$. In the latter case $\bar{s}^{F-1} \rightarrow \bar{s}^{F-2}$. Proceeding in this manner, after a finite number of steps we reach $\bar{s}^{0}=s^{* 2}$ which is a strict Nash equilibrium. If $\bar{s}_{i}^{F}$ is not a best reply to $\bar{s}_{-i}^{F}$ for $i=F+1$, then a similar argument shows that after a finite number of steps we either reach $\bar{s}^{I / 2}=s^{* 3}$ and stop, if the latter is a Nash equilibrium. Or otherwise we reach $\bar{s}^{I}=s^{* 1}$, which always is a strict Nash equilibrium.

If $c=4 K(I-1) /(I(I+2))$, then $s^{* 1}$ and $s^{* 2}$ are the unique strict Nash equilibria. Hence, if $F=0$ we are done. If $1 \leq F \leq I / 2$, then $\bar{s}_{i}^{F}$ is not the unique best reply to $\bar{s}_{-i}^{F}$ for $i=F$ since

$$
K\left(\frac{I-F}{I-F+1}-\frac{F-1}{F}\right) \geq \frac{c}{I-1}(I-2 F+1),
$$

which is fulfilled for all $1 \leq F \leq I / 2$. Hence $\bar{s}^{F} \rightarrow \bar{s}^{F-1}$ and either $F-1=0$ and we are done or by the same argument as above $\bar{s}^{F-1} \rightarrow \bar{s}^{F-2}$. Again, after a finite number of steps we reach the strict Nash equilibrium $\bar{s}^{0}=s^{* 2}$.

Now let $s^{*}$ be an arbitrary strategy profile. If $s^{*}$ is a strict Nash equilibrium we are done. Otherwise, we construct a path from $s^{*}$ to some $\bar{s}^{F}$ in the best reply graph by defining $s^{0}, s^{1}, \ldots, s^{I}$, as follows: $s^{0}=s^{*}$ and $s^{i}=\left(s_{i}, s_{-i}^{i-1}\right)$ for $i=1, \ldots, I$, where $s_{i}$ is a best reply of $i$ to $s_{-i}^{i-1}$ and $s_{i}=0$ only if 0 is the unique best reply. By construction, $s_{i}^{i} \neq 0$ for all $i$ since $c<K(I-1) / I$. Let $j \geq 1$ be minimal such that $s_{j}^{j}=2$, i.e. $s_{i}^{i}=1$ for all $i<j$. Then it is straightforward to see that $s_{i}^{i}=2$ for all $i=j+1, \ldots, I$. Hence $s^{I}=\bar{s}^{j-1}$ and we are done by the first part of the proof.

Proof of Lemma 3.2. Let $\mathcal{G}$ be the graph with vertices $\left\{h_{k}\right\}, k=1,2,3$, and directed edges $\left(\left\{h_{k}\right\},\left\{h_{l}\right\}\right)$ with weight $r_{k l}=\min _{\left(h^{1}, \ldots, h^{\tau}\right)} r\left(h^{1}, h^{2}\right)+r\left(h^{2}, h^{3}\right)+$ $\cdots+r\left(h^{\tau-1}, h^{\tau}\right)$, where the minimum is taken over all directed paths $\left(h^{1}, \ldots, h^{\tau}\right)$ with $h^{1}=h_{k}$ and $h^{\tau}=h_{l}$. By symmetry, $r_{13}=r_{23}=r^{*}, r_{31}=r_{32}=\tilde{r}$ and $r_{12}=r_{21}=: r$. Define a tree rooted at vertex $\left\{h_{k}\right\}$ to be a spanning tree in $\mathcal{G}$ such that from every vertex $\left\{h_{l}\right\}$ different from $\left\{h_{k}\right\}$ there is a unique directed path 
from $\left\{h_{l}\right\}$ to $\left\{h_{k}\right\}$. The resistance of a rooted tree is defined to be the sum of the resistances on the edges that compose it. Finally, the stochastic potential $\gamma_{k}$ of the recurrent communication class $\left\{h_{k}\right\}$ is defined to be the minimum resistance over all trees rooted at $\left\{h_{k}\right\}$.

As Young (1993) (Theorem 2, resp. Theorem 4 in the Appendix) has shown, the stochastically stable states of adaptive play $P^{\varepsilon}$ are the states contained in the recurrent communication classes of $P^{0}$ with minimum stochastic potential. By Theorem 3.1 the recurrent communication classes of the process $P^{0}$ are singletons and contain the conventions as their unique element. Hence, it remains to determine the stochastic potential of each class $\left\{h_{k}\right\}, k=1,2,3$. Since it is obviously true that $r \geq \max \left\{r^{*}, \tilde{r}\right\}$, we find that $\gamma_{1}=\gamma_{2}=r^{*}+\tilde{r}$ and $\gamma_{3}=2 r^{*}$, which immediately implies the claim of the lemma.

Proof of Theorem 3.3. By Lemma 3.2, $h_{1}$ and $h_{2}$ are the unique stochastically stable states for $c \leq 4 K(I-1) /(I(I+2))$, since in this case $\tilde{r}=0<r^{*}$. Hence, it remains to consider the case $c>4 K(I-1) /(I(I+2))$, for which $s^{* 1}, s^{* 2}$ and $s^{* 3}$ are all strict Nash equilibria. For $0 \leq F \leq I$ let $\bar{s}^{F} \in \prod_{i \in I} S_{i}$ be defined as in (6) and let $F^{*}$ be the minimal $F \geq 1$ such that $s_{i}=1$ is a best reply to $\bar{s}_{-i}^{F}$ for $i=F$. We obtain

$$
F^{*}=\left\lceil\frac{I+1}{2}-\sqrt{\left(\frac{I+1}{2}\right)^{2}-\frac{K(I-1)}{c}}\right\rceil \cdot{ }^{17}
$$

Similarly, let $\tilde{F}$ be the minimal $F \geq 1$ such that $s_{i}=1$ is a best reply to $s_{-i}^{I / 2+F}$ for $i=I / 2+F$. Then

$$
\tilde{F}=\left\lceil\frac{1}{2}+\sqrt{\left(\frac{I+1}{2}\right)^{2}-\frac{K(I-1)}{c}}\right\rceil .
$$

Observe that $F^{*}, \tilde{F} \geq 2$. Assume now that the economy is in state $h_{2}$. Any path from $h_{2}$ to $h_{3}$ has to reach a state $h$ with the following property (P):

If $s$ is one of the $n$ right-most elements of $h$, then there exists $F=$ $F(s), F^{*} \leq F \leq I / 2$, such that $s_{i}=1$ for all $i \leq F$ and $s_{i}=2$ for all $i \geq I / \overline{2}+1 .^{1 \overline{8}}$

We will show that there exists a path of zero resistance from $h$ to $h_{3}$. To this end, let $\left(s^{1}, \ldots, s^{n}\right)$ be the sample of the last $n$ observations in $h$ and let $F^{l}=F\left(s^{l}\right)$ as defined in property (P). Let $s_{i}$ be a best reply of $i$ to this sample. Then $s_{i}=2$ for all $i \geq I / 2+1$ (otherwise going to market 1 would also be a best reply to $s_{-i}^{* 3}$ ). Moreover, by definition of $F^{*}$ it follows that $s_{i}=1$ for all $i \leq \min _{l} F^{l}$ and $s_{i}=1$ for $i=\min _{l} F^{l}+1$, whenever $\min _{l} F^{l}<I / 2$. Let $h^{0}=h$ and for all $l \geq 1$ let $h^{l}$ be the successor of $h^{l-1}$, such that if $s^{l}$ is the last element of $h^{l}$, then for all $i, s_{i}^{l}$ is a best reply of $i$ to the last $n$ observations in $h^{l-1}$. Given our observation above, if $F^{*}=I / 2$ or $F^{*}+1=I / 2$, then $s^{l}=s^{* 3}$ for all $l \geq 1$, and therefore $h^{m}=h_{3}$

17 By $\lceil x\rceil$ we denote the smallest integer larger or equal to $x \in \mathbb{R}$.

18 For example, $h_{3}$ itself has this property. 
and we are done. If $F^{*}+1<I / 2$, then by the same reasoning as above $s_{i}^{l}=1$ for all $i \leq F^{*}+2$ and $l \geq n+1$. Again, if $F^{*}+2=I / 2$, then $s^{l}=s^{* 3}$ for all $l \geq n+1$ and it follows that $h^{n+m}=h_{3}$. If $F^{*}+2<I / 2$ we repeat the argument. In any case we see that there exists $N \geq 1$ such that $h^{N}=h_{3}$, i.e. there is a path of zero resistance from $h$ to $h_{3}$.

This implies, that the minimum resistance over all paths from $h_{2}$ to $h_{3}, r^{*}$, can be characterized as the minimum total number of mistakes such that, starting from $h_{2}$ the adaptive play process reaches a state $h$ having property (P). Therefore, $r^{*}$ is non-decreasing in $F^{*}$. We will now construct such a path and determine its resistance, which will give an upper bound on $r^{*}$. Starting from $h_{2}$ let the players $i=1, \ldots, F^{*}$, choose $s_{i}=1, n$ times in succession (either as a best reply or by mistake) and let any $i>F^{*}$ sample from the last $n$ observations in any history and play a best reply. In this way we obtain a path of histories $h^{0}, h^{1} \ldots, h^{n}$, with $h^{0}=h_{2}$ and such that for the last element $s^{l}$ of $h^{l}(l=1, \ldots, n)$ it is true that $s_{i}^{l}=1$ for all $i \leq F^{*}$ and $s_{i}^{l}$ is a best reply to the last $n$ observations in $h^{l-1}$ for all $i \geq F^{*}+1$. Then $s_{i}^{l}=2$ for all $i \geq I / 2+1$ and all $l=1, \ldots, n$. Hence, $h^{n}$ has property (P) and the resistance of the path from $h_{2}$ to $h^{n}$, and hence from $h_{2}$ to $h_{3}$, is less than or equal to $n F^{*}$. Since starting from $h_{2}$ one obviously needs at least $F^{*}$ mistakes to reach $h_{3}$, we conclude that

$$
F^{*} \leq r^{*} \leq n F^{*}
$$

In a similar way we obtain that $\tilde{r}$ is non-decreasing in $\tilde{F}$ and

$$
\tilde{F} \leq \tilde{r} \leq n \tilde{F} .
$$

Let $I>4 n$. Since $F^{*}$ is non-increasing in $c$ and $r^{*}$ is non-decreasing in $F^{*}$ it follows that $r^{*}$ is non-increasing in $c$. Similarly, since $\tilde{F}$ is non-decreasing in $c$ and $\tilde{r}$ is non-decreasing in $\tilde{F}$ it follows that $\tilde{r}$ is non-decreasing in $c$. If $c$ is close to $K(I-1) / I$, then $F^{*}=2$ and $\tilde{F}=I / 2$. Hence, it follows that $r^{*} \leq 2 n$ and $\tilde{r} \geq I / 2$. Since $I>4 n$ we conclude that $\tilde{r}>r^{*}$. On the other hand, if $c$ is close to $4 \bar{K}(I-1) /(I(I+2))$, then $F^{*}=I / 2$ and $\tilde{F}=2$. In this case $r^{*} \geq I / 2$ and $\tilde{r} \leq 2 n$ and hence $r^{*}>\tilde{r}$ since $I>4 n$. Thus, given the monotonicity property of $r^{*}$ and $\tilde{r}$ we obtain the existence of some $c_{1}^{*}, c_{2}^{*} \in(4 K(I-1) /(I(I+2)), K(I-1) / I)$, $c_{1}^{*} \leq c_{2}^{*}$, such that $\tilde{r}<r^{*}$ for $c<c_{1}^{*}, \tilde{r}>r^{*}$ for $c>c_{2}^{*}$ and $\tilde{r}=r^{*}$ for all $c \in\left(c_{1}^{*}, c_{2}^{*}\right)$ in case $c_{1}^{*}<c_{2}^{*}$. This proves the theorem.

Proof of Theorem 3.4. We fix the sample size $n$. In the following we write $F^{*}, \tilde{F}, r^{*}$ and $\tilde{r}$ as functions of $I$. By the definition of $F^{*}$ in (7) it follows that

$$
F^{*}(I) \leq \frac{I+3}{2}-\sqrt{\left(\frac{I+1}{2}\right)^{2}-\frac{K(I-1)}{c}}=: g(I) .
$$

If $(c+K) / c$ is not an integer, let $\delta>0$ be such that $\lceil(c+K) / c\rceil>(c+K) / c+\delta$. Otherwise, if $(c+K) / c$ is an integer, let $0<\delta<1$ be arbitrary. We will show that there exists $I_{0}=I_{0}(c, K)$ such that

$$
g(I) \leq \frac{c+K}{c}+\delta,
$$


for all $I \geq I_{0}$. For $I$ sufficiently large (11) is equivalent to

$$
\delta I \geq\left(\frac{c+K}{c}+\delta\right)^{2}-3\left(\frac{c+K}{c}+\delta\right)+2-\frac{K}{c},
$$

which follows from a straightforward computation. Clearly, there exists $I_{0}=$ $I_{0}(c, K)$ such that (12) and hence (11) is satisfied for all $I \geq I_{0}$. By the choice of $\delta$ this proves that $F^{*}(I) \leq(c+K) / c$, since $F^{*}(I)$ is an integer. Hence, by (9) it follows that $r^{*}(I) \leq n(c+K) / c$ for $I \geq I_{0}$.

By (10), in order to prove that $\lim _{I \rightarrow \infty} \tilde{r}(I)=\infty$ it suffices to show that $\lim _{I \rightarrow \infty} \tilde{F}(I)=\infty$. By the definition of $\tilde{F}$ in (8) it follows that

$$
\tilde{F}(I) \geq-\frac{1}{2}+\sqrt{\left(\frac{I+1}{2}\right)^{2}-\frac{K(I-1)}{c}}
$$

and it is immediately seen that the right hand side of this inequality goes to infinity for $I \rightarrow \infty$. This proves the first part of the theorem.

Hence, for all $0<c<K$ there exists $I(c)$ such that $\tilde{r}(I)>r^{*}(I)$ for all $I \geq I(c)$. By Theorem 3.3 this implies $c \geq c_{2}^{*}(I)$ for all $I \geq I(c)$. Since $c$ was arbitrary it follows that $\lim _{I \rightarrow \infty} c_{2}^{*}(I)=0$ and therefore $\lim _{I \rightarrow \infty} c_{1}^{*}(I)=$ $\lim _{I \rightarrow \infty} c_{2}^{*}(I)=0$.

Proof of Theorem 3.5. By Ellison (2000) (Lemma 6), $W(h, \varepsilon)=O\left(\varepsilon^{-C R}\right)$, where $C R$ is the coradius of the basin of attraction of the recurrent class $\left\{h_{3}\right\}$. $C R$ is defined by $C R=\max _{h \neq h_{3}} \min _{\left(h^{1}, \ldots, h^{t}\right)} r\left(h^{1}, h^{2}\right)+r\left(h^{2}, h^{3}\right)+\cdots+r\left(h^{t-1}, h^{t}\right)$, where the minimum is taken over all paths $\left(h^{1}, \ldots, h^{t}\right)$ with $h^{1}=h, h^{t}=h_{3}$ and $h^{\tau} \neq h^{\tau^{\prime}}$ for all $\tau, \tau^{\prime} \in\{1, \ldots, t\}, \tau \neq \tau^{\prime}$. Hence, $C R=r^{*}$ and the claim follows from Theorem 3.4.

Proof of Theorem 3.6. Since $F^{*}$ is non-decreasing and $\tilde{F}$ is non-increasing in $K$ it follows that $r^{*}$ is non-decreasing and $\tilde{r}$ is non-increasing in $K$ for fixed cost $c$. Hence, the endpoints of the interval $\left(c_{1}^{*}, c_{2}^{*}\right)$ on which the graphs of $r^{*}$ and $\tilde{r}$ intersect are non-decreasing in $K$. The claim then immediately follows if we recall that $K=\gamma \sigma^{2} \sigma_{e}^{2} / 2$.

\section{References}

Alesina, A., Spolaore, E.: On the number and size of nations. Quart J Econ 112, 1027-1056 (1997)

Alós-Ferrer, C., Kirchsteiger, G.: Does learning lead to coordination in market clearing institutions? Working Paper 0319, Department of Economics, University of Vienna (2003)

Bettzüge, M. O., Hens, T.: An evolutionary approach to financial innovation. Rev Econ Stud 68, 493-522 (2001)

Economides, E., Siow, A.: The division of markets is limited by the extent of liquidity (spatial competition with externalities). Am Econ Rev 78, 108-121 (1988)

Ellison, G.: Learning, local interaction, and coordination. Econometrica 61, 1047-1071 (1993)

Ellison, G.: Basins of attraction, long-run stochastic stability, and the speed of step-by-step evolution. Rev Econ Stud 67, 17-45 (2000)

Güth, S., Ludwig, S.: Innovation and information - a study of financial markets' evolution. Ph.D. thesis, Bielefeld University (2000) 
Kandori, M. Mailath, G.J., Rob, R.: Learning, mutation, and long run equilibria in games. Econometrica 61, 29-56 (1993)

O'Hara, M.: Market microstructure theory. Cambridge: Blackwell 1995

Pagano, M.: Endogenous market thinness and stock price volatility. Rev Econ Stud 56, 269-287 (1989a)

Pagano, M.: Trading volume and asset liquidity. Quart J Econ 104, 255-274 (1989b)

Young, H.P.: The evolution of conventions. Econometrica 61, 57-84 (1993) 